\section{Determinação dos metabolismos lático e alático da capacidade anaeróbia por meio do consumo de oxigênio}

\section{Determination of lactic and alactic metabolisms of the anaerobic capacity using oxygen uptake}

\author{
Rodrigo Poles Urso \\ Marcos David Silva-Cavalcante ${ }^{1,2}$ \\ Carlos Rafaell Correia-Oliveira ${ }^{1,2}$ \\ Salomão Bueno ${ }^{1}$ \\ Mayara Vieira Damasceno \\ Adriano Eduardo Lima-Silva ${ }^{2}$ \\ Rômulo Bertuzzi ${ }^{1}$
}

Resumo - Tem sido sugerido que a participação do metabolismo anaeróbio alático (MAA) pode ser estimada a partir do cálculo da fase rápida do excesso do consumo de oxigênio

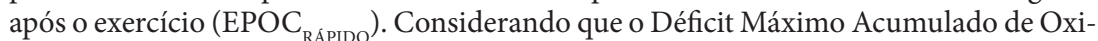
gênio (MAOD) representa a quantidade total de energia transferida pelos metabolismos anaeróbios, o objetivo desse estudo foi analisar se o método de subtrair o $\mathrm{EPOC}_{\mathrm{RAPIDO}}$ do MAOD ( MAOD $\left._{\mathrm{LA}-1}\right)$ proporciona uma estimativa satisfatória do metabolismo anaeróbio lático (MAL). Para esse fim, o MAOD $\mathrm{LA-1}_{1}$ foi comparado ao método capaz de expressar em equivalente de oxigênio a energia oriunda do acúmulo de lactato no sangue $\left(\mathrm{MAOD}_{\mathrm{LA}-2}\right)$. Nove homens adultos ativos foram submetidos a quatro sessões experimentais: 1) um teste progressivo até a exaustão em um cicloergômetro para a mensuração do consumo máximo de oxigênio $\left(\mathrm{VO}_{2 \max }\right)$ e da potência externa correspondente ao $\mathrm{VO}_{2 \max }\left(\mathrm{WVO}_{2 \max }\right) ; 2$ e 3$)$ seis testes de cargas constantes ( 3 testes por sessão) com intensidades abaixo da $\mathrm{WVO}_{2 \max } ; 4$ ) um teste de carga constante com a intensidade equivalente a $110 \%$ da $\mathrm{WVO}_{2 \max }$. O principal achado foi que os dois métodos $\left(\mathrm{MAOD}_{\mathrm{LA}_{-1}}\right.$ e $\left.\mathrm{MAOD}_{\mathrm{LA}-2}\right)$ empregados na estimativa da contribuição do MAL no MAOD geraram valores estatisticamente similares ( $p>0,05)$. Além disso, os valores percentuais do $\mathrm{MAOD}_{\mathrm{LA}-1}$ (representando o MAL) e do $\mathrm{EPOC}_{\text {RÁPIDO }}$ (representando o MAA) foram de aproximadamente $78 \%$ e 22\%, respectivamente. Logo, os procedimentos propostos na presente investigação podem auxiliar futuros trabalhos que porventura objetivem fragmentar as contribuições dos componentes anaeróbio do MAOD.

Palavras-chave: Bioenergética; Lactato; Oxigênio.

Abstract - It has been suggested that the participation of alactic anaerobic metabolism (AAM) in physical activity can be estimated by calculating the fast component of excess post-exercise oxygen consumption $\left(E P O C_{F A S T}\right)$. Considering that maximal accumulated oxygen deficit (MAOD) represents the total amount of energy transferred by anaerobic metabolisms, this study aimed to analyze whether subtracting $E P O C_{F A S T}$ from MAOD $\left(M A O D_{L A-1}\right)$ provides a satisfactory estimate of lactic anaerobic metabolism (LAM). For this purpose, $M A O D_{L A-1}$ was compared with the method by which the energy derived from blood lactate accumulation $\left(M A O D_{L A-2}\right)$ is expressed as oxygen equivalent. Nine active adult men were submitted to four experimental sessions: 1) a maximal incremental exercise test until exhaustion on a cycle ergometer to measure the maximal oxygen consumption $\left(\mathrm{VO}_{2} \mathrm{max}\right)$ and the external power corresponding to $\mathrm{VO}_{2} \max \left(\mathrm{WVO}_{2}\right.$ max); 2) and 3) six constant-workload tests (3 per session) at intensities below $W V O$ max; 4) one constant-workload test at an intensity corresponding to $110 \%$ of $\mathrm{WVO}_{2}$ max. The main finding was that both methods $\left(\mathrm{MAOD}_{L A-1} e\right.$ $\left.M A O D_{L A-2}\right)$ that were used to estimate the contribution of LAM in MAOD resulted in similar statistical values $(p>0.05)$. In addition, percentage values of $M A O D_{L A-1}($ representing $M A L)$ and $E P O C_{F A S T}$ (representing $\left.A A M\right)$ were approximately 78 and $22 \%$, respectively. Thus, the procedure proposed in this paper may be of interest to future studies on the

Key words: Bioenergetics; Lactate; Oxygen.
1 Universidade de São Paulo. Escola de Educação Física e Esporte. Grupo de Estudos em Desempenho Aeróbio. São Paulo, SP. Brasil.

2 Universidade Federal de Alagoas. Faculdade de Nutrição. Grupo de Pesquisa em Ciências do Esporte. Alagoas, AL. Brasil.

Recebido em 25/06/12 Revisado em 12/01/13 Aprovado em 17/03/13 


\section{INTRODUÇÃOO}

Acredita-se que, durante o exercício cíclico de alta intensidade, o metabolismo aeróbio exerce a sua predominância a partir do trigésimo segundo ${ }^{1}$. Por outro lado, nos instantes iniciais dos exercícios físicos de alta intensidade e com curta duração (<30s), a sustentação dos processos que requerem energia durante a contração muscular ocorre, preferencialmente, por vias que não necessitam de oxigênio $\left(\mathrm{O}_{2}\right)$, denominadas de metabolismo anaeróbio ${ }^{2}$. Esse metabolismo, por sua vez, é subdividido em metabolismo anaeróbio alático (MAA) e metabolismo anaeróbio lático (MAL). O MAA se refere à hidrólise dos estoques de adenosina trifosfato (ATP) e de fosfocreatina (CP), ao passo que o MAL se refere à degradação parcial da glicose, resultando na formação de ácido lático ${ }^{2,3}$.

A capacidade anaeróbia (CAN), definida como a quantidade total de energia transferida pelos metabolismos anaeróbios, durante a execução de um exercício de alta intensidade ${ }^{3}$, pode ser estabelecida com precisão pela análise direta dos substratos do metabolismo anaeróbio, os quais são obtidos por meio de biópsia muscular antes e após o exercício físico ${ }^{4}$. No entanto, em virtude da característica invasiva dessa técnica e da dificuldade em se estimar a massa muscular envolvida, alguns estudos têm empregado o Déficit Máximo Acumulado de Oxigênio (MAOD) na determinação da contribuição anaeróbia em diversas tarefas esportivas ${ }^{1,5}$.

Alguns estudos têm apresentado limitações acerca do $\mathrm{MAOD}^{6-7}$. Por exemplo, Buck e Mcnaughton ${ }^{7}$ demonstraram que a redução do número de testes submáximos para o cálculo do MAOD é capaz de interferir nos resultados, sugerindo que o MAOD é dependente do tipo de protocolo utilizado. Entretanto, a validade desse teste tem sido demonstrada a partir de diferentes perspectivas ${ }^{8-9}$. Do ponto de vista metodológico, o cálculo do MAOD é baseado na mensuração do consumo de oxigênio $\left(\mathrm{VO}_{2}\right) \mathrm{em}$ múltiplos testes de cargas constantes. Inicialmente, a relação linear entre $\mathrm{VO}_{2}$-carga dos testes submáximos é determinada para estimar a demanda metabólica do exercício supramáximo. Em seguida, o MAOD é calculado pela diferença entre a demanda metabólica estimada e o $\mathrm{VO}_{2}$ acumulado durante o exercício supramáximo, o qual deve ter a duração entre 2 e 6 minutos ${ }^{10}$.

A partir de dados obtidos por biópsia muscular, observou-se que as participações do MAL e do MAA no MAOD são em torno de 75\% e 25\%, respectivamente ${ }^{7}$. Contudo, ao passo que o valor do MAOD pode variar, por exemplo, a partir de efeitos de suplementação ${ }^{11,12}$, é plausível pressupor que essa variação pode ocorrer em razão da variação da contribuição do MAA e do MAL. Nesse sentido, parece ser importante a realização de estudos que proponham outros métodos mais simples do que a biópsia muscular e que também sejam capazes de estimarem a participação dos componentes anaeróbios no MAOD, com a finalidade de analisar uma possível variação na magnitude da participação em cada um deles após algum tipo de intervenção. 
Acredita-se que durante a fase inicial do período de recuperação de um exercício o restabelecimento dos estoques da $\mathrm{CP}$ ocorre por processos metabólicos que dependem do $\mathrm{VO}_{2}{ }^{13,14}$. Por sua vez, di Prampero e Ferret$\mathrm{ti}^{14}$ apresentaram um método capaz de expressar em equivalente de $\mathrm{O}_{2}$ a energia oriunda do acúmulo de lactato no sangue $\left(\Delta\left[\mathrm{La}^{-}\right]\right)$. Logo, diversos trabalhos empregaram a fase rápida do excesso do consumo de oxigênio após o exercício (EPOC ${ }_{\text {RÁPIDO }}$ ) e a concentração sanguínea de lactato ([ $\mathrm{La}^{-}$ ]) para estimarem as participações do MAA e MAL, respectivamente ${ }^{15,16}$.

Nesse sentido, ao considerarmos que o EPOC $_{\text {RÁPIDO }}$ reflete a participação do MAA, é possível suspeitar que a diferença dessa medida pelo MAOD possa representar a participação do MAL. Nesse caso, sugere-se que informações acerca dos MAA e MAL possam ser proporcionadas, utilizando-se apenas a mensuração do $\mathrm{VO}_{2}$, sem a medida de lactato. Portanto, o objetivo desse estudo foi analisar se a subtração do EPOC $_{\text {RÁPIDO }}$ do MAOD proporciona uma estimativa satisfatória do MAL. Com base nos referenciais teóricos e procedimentais empregados nos estudos supracitados, a nossa hipótese foi que a porção do MAL no MAOD pode ser determinada subtraindo-se o $\mathrm{EPOC}_{\mathrm{RÁPIDO}}$ do MAOD $\left(\mathrm{MAOD}_{\mathrm{LA}-1}\right)$. Além disso, os valores do $\mathrm{MAOD}_{\mathrm{LA}-1}$ seriam similares aos cálculos propostos por di Prampero e Ferretti ${ }^{14}$ para a determinação do MAL mediante a análise das concentrações sanguíneas de lactato $\left(\mathrm{MAOD}_{\mathrm{LA}-2}\right)$.

\section{PROCEDIMENTOS METODOLÓGICOS}

\section{Amostra}

Nove indivíduos fisicamente ativos do sexo masculino (idade: $23 \pm 4$ anos, estatura: $176,4 \pm 6,8 \mathrm{~cm}$, massa corporal: $72,4 \pm 8,2 \mathrm{~kg}$ e percentual de gordura corporal: $12,0 \pm 4,5 \%$ ), aparentemente saudáveis e já familiarizados com exercício exaustivo, participaram voluntariamente desse estudo após a leitura e assinatura do termo de consentimento livre e esclarecido. Todos os sujeitos estavam isentos de tratamentos farmacológicos, não eram fumantes e estavam livres de qualquer tipo de distúrbio neuromuscular ou cardiovascular. Os procedimentos adotados no presente estudo foram previamente aprovados pelo Comitê de Ética em Pesquisa da Escola de Educação Física e Esporte da Universidade de São Paulo (Protocolo de Pesquisa n. 2006/06).

\section{Desenho experimental}

Todos os sujeitos foram submetidos a quatro sessões experimentais, as quais tiveram o intervalo mínimo de 72 horas e máximo de três semanas. $\mathrm{Na}$ primeira sessão, os sujeitos realizaram um teste progressivo até a exaustão em um cicloergômetro para a mensuração do consumo máximo de oxigênio ( $\left.\mathrm{VO}_{2} \max \right)$ e da potência externa correspondente ao $\mathrm{VO}_{2} \max \left(\mathrm{WVO}_{2} \max \right)$. Na segunda e na terceira sessões, os sujeitos foram submetidos a seis testes de cargas constantes ( 3 testes por sessão) com intensidades abaixo da $\mathrm{WVO}_{2}$ max. Na quarta sessão, foi realizado um teste de carga constante 
com a intensidade equivalente a $110 \%$ da $\mathrm{WVO}_{2}$ max. A ordem das sessões 2-3, bem como a dos testes sub- $\mathrm{VO}_{2} \max$ foi estabelecida aleatoriamente. Os testes foram realizados com a temperatura ambiente semelhante (20$24^{\circ} \mathrm{C}$ ) e duas horas após a última refeição. Os sujeitos foram solicitados a não praticarem exercícios físicos extenuantes e a não ingerirem álcool nas 48 horas que antecederam as coletas dos dados. No intuito de evitar possíveis influências ergogênicas e do ritmo circadiano, todos os testes foram realizados no mesmo período do dia e os sujeitos foram instruídos a não consumirem substâncias que possuíssem cafeína nas 48 horas que precediam os testes.

\section{Medidas antropométricas}

As medidas antropométricas foram realizadas por um único avaliador, conforme os procedimentos sugeridos por Norton e Olds ${ }^{17}$. A massa corporal foi medida por uma balança eletrônica com precisão de $0,1 \mathrm{~kg}$ (Filizola, modelo ID 1500, São Paulo, Brasil) e a estatura foi mensurada com um estadiômetro de madeira com precisão de $0,1 \mathrm{~cm}$. As dobras cutâneas foram medidas em quatro pontos anatômicos (tríceps, suprailíaca, abdominal e coxa) por meio de um compasso da marca Harpenden (West Sussex, UK). A mediana dos valores dessas medidas, as quais foram realizadas três vezes e ao lado direito do corpo, foi empregada na estimativa da composição corporal. A densidade corporal foi estimada pela equação generalizada de Jackson e Pollock ${ }^{18}$, ao passo que o percentual de gordura foi estabelecido pela equação de Brozek et al. ${ }^{19}$.

\section{Determinação do consumo máximo de oxigênio}

O teste incremental até a exaustão foi realizado em um cicloergômetro eletromagnético de membros inferiores (Godart-Holland, LANNOY). A altura do selim foi individualmente ajustada, tendo como referência a extensão quase total dos joelhos em um ciclo completo do pedal. Antes do início do teste, os participantes permaneceram cinco minutos sentados no cicloergômetro para o estabelecimento da linha de base do $\mathrm{VO}_{2}\left(\mathrm{VO}_{2 \mathrm{LB}}\right)$, a qual foi determinada a partir da média aritmética do $\mathrm{VO}_{2}$ nos trinta segundos finais desse período. Após o aquecimento de 3 minutos com apenas a resistência inercial do equipamento, os sujeitos se exercitaram com o ritmo em $60 \mathrm{rpm}$ e com o incremento da intensidade de $30 \mathrm{~W} \cdot \mathrm{min}^{-1}$. O teste foi interrompido quando a cadência do pedal era menor que $50 \mathrm{rpm}$. Durante todo o teste as trocas gasosas e a frequência cardíaca (FC) foram mensuradas respiração a respiração e a cada batimento, respectivamente. Amostras de sangue foram coletas imediatamente após o teste, no segundo e no terceiro minutos da recuperação para mensuração das concentrações sanguíneas de lactato de pico ([La]pico), ao passo que a frequência cardíaca máxima (FCmax) foi estabelecida pelo maior valor medido ao final do teste. $\mathrm{O} \mathrm{VO}_{2} \max$ foi determinado quando, pelo menos, três dos seguintes critérios foram obtidos: 1) exaustão voluntária do sujeito; 2) aumento do $\mathrm{VO}_{2}$ menor que $2,1 \mathrm{ml} \cdot \mathrm{kg}^{-1} \cdot \mathrm{min}^{-1}$ mediante o incremento da intensidade; 
3) razão de trocas respiratórias maiores que 1,10 ; 4) concentração de lactato sanguíneo após o teste maior que $8,0 \mathrm{mmol}^{-1}$; 5) FCmax predita pela idade (220-idade $)^{20}$. A WVO $\max$ foi estabelecida como a potência externa máxima em que o $\mathrm{VO}_{2} \max$ foi alcançado.

\section{Testes submáximos com cargas constantes}

O cicloergômetro, o ajuste da altura do selim, o ritmo do pedal, o aquecimento, o critério de interrupção e a mensuração do $\mathrm{VO}_{2}$ nos exercícios com cargas constantes foram idênticos aos empregados no teste progressivo até a exaustão. Os sujeitos se exercitaram por dez minutos, ou até a exaustão voluntária, em seis testes de intensidades abaixo da $\mathrm{WVO}_{2} \max (40-90 \% \mathrm{da}$ $\left.\mathrm{WVO}_{2} \max \right)$. O período de recuperação entre essas tarefas foi de aproximadamente dez minutos, ou até atingir o valor individual do $\mathrm{VO}_{2 \mathrm{LB}}$. A média do $\mathrm{VO}_{2}$, no último minuto dessas tarefas, foi utilizada para representar o valor de pico do $\mathrm{VO}_{2}$ nessas tarefas. O pico da $\mathrm{FC}$ foi medido ao final dos testes. Além disso, amostras contendo $25 \mu$ de sangue foram coletadas do lóbulo da orelha antes de cada teste ([La-]repouso), imediatamente após o término, no terceiro, no quinto e no sétimo minutos de recuperação para a mensuração das $\left[\mathrm{La}^{-}\right]$. O maior valor após o exercício foi utilizado para representar o lactato de pico.

\section{Teste supramáximo com carga constante}

No teste supramáximo, os sujeitos se exercitaram até a exaustão voluntária em uma intensidade acima da $\mathrm{WVO}_{2} \max \left(110 \%\right.$ da $\left.\mathrm{WVO}_{2} \max \right)$. Nesse caso, o cicloergômetro, o ajuste da altura do selim, o ritmo do pedal, o aquecimento, o critério de interrupção e a mensuração do $\mathrm{VO}_{2}$ foram idênticos aos empregados no teste progressivo até a exaustão. Além disso, o valor pico do $\mathrm{VO}_{2}$ e da FC, assim como as medidas das [La'] de repouso e pico, foram feitas da mesma forma que nos testes submáximos com cargas constantes.

\section{Coleta dos dados fisiológicos}

$\mathrm{O} \mathrm{VO}_{2}$ foi mensurado continuamente por meio de um analisador de gases

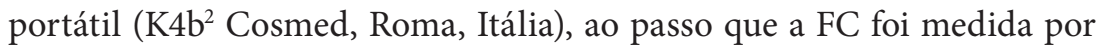
um cardiofrequencímetro (Polar, Kempele, Finlândia) acoplado a esse equipamento. Uma máscara de silicone com baixa resistência que mantém o nariz e a boca cobertos (Hans Rudolph, Kansas City, USA) foi empregada na coleta do ar utilizado nas análises da ventilação, das frações do oxigênio e do dióxido de carbono. Antecedendo cada sessão de coleta de dados, o analisador de gases foi calibrado, utilizando o ar ambiente e com um gás de composição conhecida (20,9\% de $\mathrm{O}_{2}$ e $5 \%$ de $\left.\mathrm{CO}_{2}\right)$. A calibração do fluxo de ar foi realizada com uma seringa com volume de 3 litros (Quinton Instruments, Seattle, WH). A calibragem do tempo de atraso para a análise da amostra de ar expirada foi realizada de acordo com as especificações do fabricante ( $\mathrm{K} \mathrm{b}^{2}$ instruction manual). Esse tempo de atraso é de, aproximadamente, $500 \mathrm{~ms}$ e automaticamente considerado nos cálculos das trocas gasosas. As concentrações sanguíneas de lactato foram determinadas por 
meio de um analisador automático de lactato (Yellow Springs 1500 Sport, Ohio, USA), o qual foi calibrado a cada dez análises, tendo como referência a concentração de lactato de $5 \mathrm{mmol}^{-1}{ }^{-1}$.

\section{Cálculos}

A média aritmética do $\mathrm{VO}_{2}$ nos trinta segundos finais dos exercícios sub$-\mathrm{WVO}_{2} \max$ foi plotada sobre as suas respectivas intensidades para desenvolver individualmente as equações de regressão linear. Os coeficientes angulares gerados a partir dessas equações foram empregados na estimativa da demanda de oxigênio $\left(\mathrm{VO}_{2 \mathrm{DEM}}\right)$ do exercício supra- $\mathrm{WVO}_{2} \max$ (Equação 1). O método trapezoidal foi utilizado para calcular a área integral do $\mathrm{VO}_{2}$ durante a realização do exercício supra- $\mathrm{WVO}_{2} \max$. Em seguida, o $\mathrm{VO}_{2}$ acumulado $\left(\mathrm{VO}_{2 \mathrm{ACUM}}\right)$ foi determinado a partir da exclusão do $\mathrm{VO}_{2 \mathrm{LB}}{ }^{21} \cdot \mathrm{O}$ MAOD foi estabelecido a partir da subtração do $\mathrm{VO}_{2 \mathrm{DEM}}$ sobre o $\mathrm{VO}_{2 \mathrm{ACUM}}$.

$\left.\mathrm{VO}_{2 \mathrm{DEM}}=\left[\left(b^{*} 110\right) / 60\right) \cdot t\right]$

EQUAÇÃO 1

Onde $V_{2 D E M}$ é a demanda de $0_{2}$ estimada para o exercício supra-WV0 max; 110 é a intensidade do exercício supra-WVO ${ }_{2}^{2}$ max; b é o coeficiente angular em $1 \cdot \mathrm{min}^{-1}$ gerado a partir da regressão linear estabelecida entre $\mathrm{VO}_{2}$ intensidade dos testes sub-WVO $\mathrm{max}_{2}$ t é 0 tempo de duração do exercício expresso em segundos.

Conforme descrito previamente ${ }^{22}$, na análise da resposta da cinética off do $\mathrm{VO}_{2}$ na intensidade supra- $\mathrm{WVO}_{2}$ max foi aplicado um modelo biexponencial (Equação 2) (Origin, Microcal, USA). Assim, o EPOC RÁPIDO $_{\text {foi }}$ determinado pelos termos que denotam o componente rápido do excesso do consumo após o exercício (Equação 3).

$\dot{\mathrm{VO}}_{2(t)}=\mathrm{y} 0+\left(\mathrm{A}_{1} \mathrm{e}^{-(t-\delta) / \tau_{1}}\right)+\left(\mathrm{A}_{2} \mathrm{e}^{-(t-\delta) / \tau_{2}}\right) \quad$ EQUAÇÃO2

$\mathrm{MAA}=\mathrm{A}_{1} \cdot \tau_{1}$

EQUAÇÃ0 3

Onde 1 e 2 denotam, respectivamente, o componente rápido e lento do excesso do consumo de oxigênio após 0 exercício; $\mathrm{y} 0$ = linha de base; $\mathrm{A}, \tau$ e $\delta$ são referentes à amplitude em $\mathrm{ml} \cdot \mathrm{min}-1$, à constante de tempo (s) e ao tempo de atraso (s), respectivamente.

$\mathrm{O}_{\mathrm{MAOD}}$ LA-1 foi determinado pela área remanescente do MAOD após

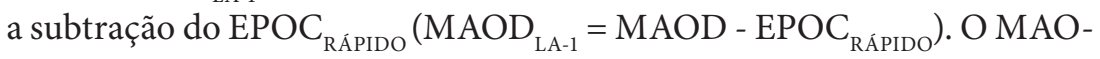
$\mathrm{D}_{\mathrm{LA}-2}$ foi calculado assumindo que $1 \mathrm{mmol}^{-1}{ }^{-1}$ dos $\Delta[\mathrm{La}]$ no exercício supra$-\mathrm{WVO}_{2}$ max equivale ao valor fixo de $3 \mathrm{ml} \mathrm{de} \mathrm{O}_{2} \cdot \mathrm{kg}^{-1}$ da massa corporal ${ }^{14}$.

\section{Análises estatísticas}

Todas as análises foram feitas utilizando o programa computadorizado SPSS (versão 13.0, Chicago, USA). A distribuição dos dados foi verificada por meio do teste de Shapiro-Wilk e apresentaram distribuição normal. Os dados foram reportados como médias e desvios padrão. A comparação entre os dois métodos adotados para se estimar a participação do MAL $\left(\mathrm{MAOD}_{\mathrm{LA}-1}\right.$ e $\left.\mathrm{MAOD}_{\mathrm{LA}-2}\right)$ no MAOD foi realizada por meio do teste $t$ de Student para dados pareados. O nível de concordância entre os dois métodos foi testado por meio da plotagem de Bland-Altman ${ }^{23}$. Além disso, a 
correlação entre os métodos foi verificada através do teste de correlação de Pearson. O nível de significância adotado para todas as análises foi de $5 \%(\mathrm{p}<0,05)$.

\section{RESULTADOS}

As variáveis analisadas no teste progressivo até a exaustão estão presentes na tabela 1 , ao passo que a tabela 2 apresenta os dados obtidos no exercício supra- $\mathrm{WVO}_{2} \max$.

Tabela 1. Variáveis analisadas no teste progressivo até a exaustão.

\begin{tabular}{lc}
\hline $\mathrm{VO}_{2 \mathrm{LB}}\left(\mathrm{l} \cdot \mathrm{min}^{-1}\right)$ & $0,28 \pm 0,57$ \\
$\mathrm{VO}_{2} \max \left(\mathrm{l} \cdot \mathrm{min}^{-1}\right)$ & $3,0 \pm 0,5$ \\
$\mathrm{VO}_{2} \max \left(\mathrm{ml} \cdot \mathrm{kg}^{-1} \cdot \mathrm{min}^{-1}\right)$ & $41,5 \pm 5,7$ \\
$\mathrm{WVO}_{2} \max (\mathrm{W})$ & $250 \pm 40$ \\
$\mathrm{R}$ & $1,29 \pm 0,09$ \\
$\mathrm{FCmax}(\mathrm{bpm})$ & $179 \pm 9$ \\
{$\left[\right.$ La-]pico $\left(\left.\mathrm{mmol} \cdot\right|^{-1}\right)$} & $10,28 \pm 1,42$ \\
\hline
\end{tabular}

Os valores são expressos em médias \pm desvios padrão. $V 02_{1 B}=$ consumo de oxigênio em repouso; $V 02 \mathrm{max}=$ consumo máximo de oxigênio; WV02max = potência externa correspondente ao V02max; $R=$ razão de trocas respiratórias; $\mathrm{FCmax}=$ frequência cardíaca máxima; [La-]pico = concentrações sanguíneas de lactato de pico.

Tabela 2. Variáveis mensuradas durante 0 exercício na intensidade de 110\% da WV02max.

\begin{tabular}{|c|c|}
\hline Tempo (s) & $161 \pm 40$ \\
\hline Intensidade (W) & $276 \pm 43$ \\
\hline $\mathrm{VO}_{2}$ pico (I) & $2,71 \pm 0,49$ \\
\hline $\mathrm{VO}_{2}$ pico $\left(\mathrm{ml} \cdot \mathrm{kg}^{-1} \cdot \mathrm{min}^{-1}\right)$ & $37,78 \pm 6,34$ \\
\hline FCpico (bpm) & $174 \pm 13$ \\
\hline$[\mathrm{La}-]$ pico $\left(\mathrm{mmol} \cdot \mathrm{l}^{-1}\right)$ & $10,89 \pm 1,20$ \\
\hline [La-]repouso $\left(\mathrm{mmol} \cdot \mathrm{l}^{-1}\right)$ & $0,73 \pm 0,15$ \\
\hline Demanda de $\mathrm{O}_{2}(\mathrm{I})$ & $8,53 \pm 2,24$ \\
\hline Consumo de $\mathrm{O}_{2}$ acumulado (I) & $5,50 \pm 2,18$ \\
\hline MAOD (I) & $3,03 \pm 0,62$ \\
\hline $\mathrm{EPOC}_{\text {RÁPIDO }}(\mathrm{I})$ & $0,98 \pm 0,45$ \\
\hline
\end{tabular}

Os valores são expressos em médias \pm desvios padrão. V02pico = consumo de oxigênio de pico; FCpico $=$ frequência cardíaca de pico; [La-]pico = concentrações sanguíneas de lactato de pico; [La-]repouso = concentrações sanguíneas de lactato em repouso; $E P O C_{\text {RÁpioo }}=$ fase rápida do excesso do consumo de oxigênio após o exercício físico.

A figura 1 mostra a comparação entre a estimativa do $\mathrm{MAOD}_{\mathrm{LA}-1}$ e do $\mathrm{MAOD}_{\mathrm{LA}-2}$. Nesse caso, não foi observada diferença significante entre os dois valores $(\mathrm{p}>0,05)$.

A concordância entre os dois métodos através da plotagem de Bland-Altman está apresentada na figura 2. Nesse caso, o valor do viés foi próximo de zero. Além disso, a correlação entre os dois métodos foi alta $(\mathrm{r}=0,91$, $\mathrm{p}<0,01)$. 


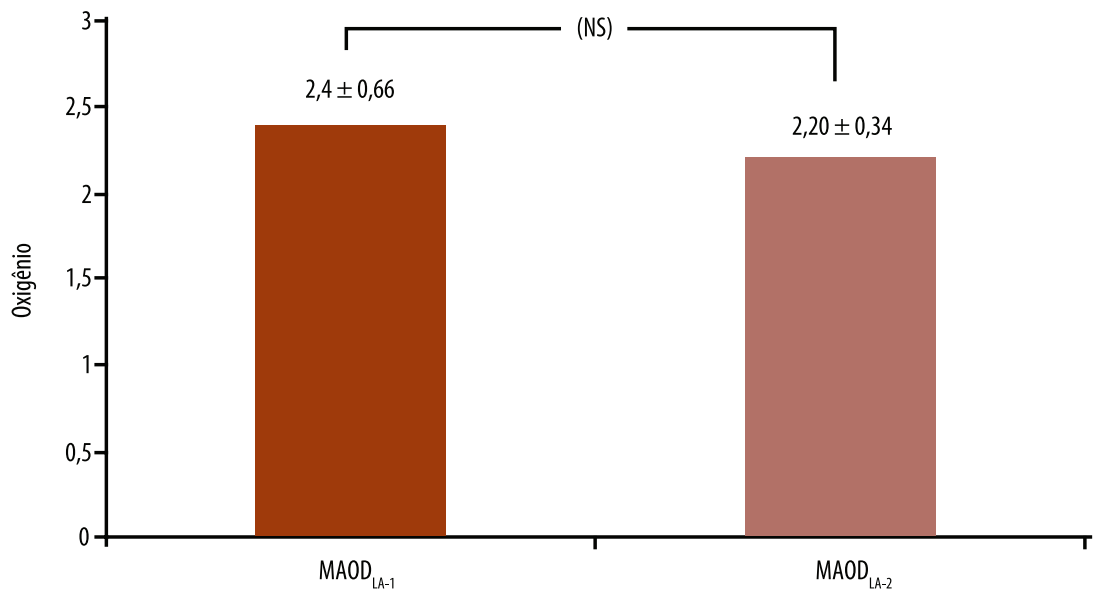

Figura 1. Comparação da estimativa do MAODLA-1 e do MAODLA-2 no MAOD.

NS = diferença não significante

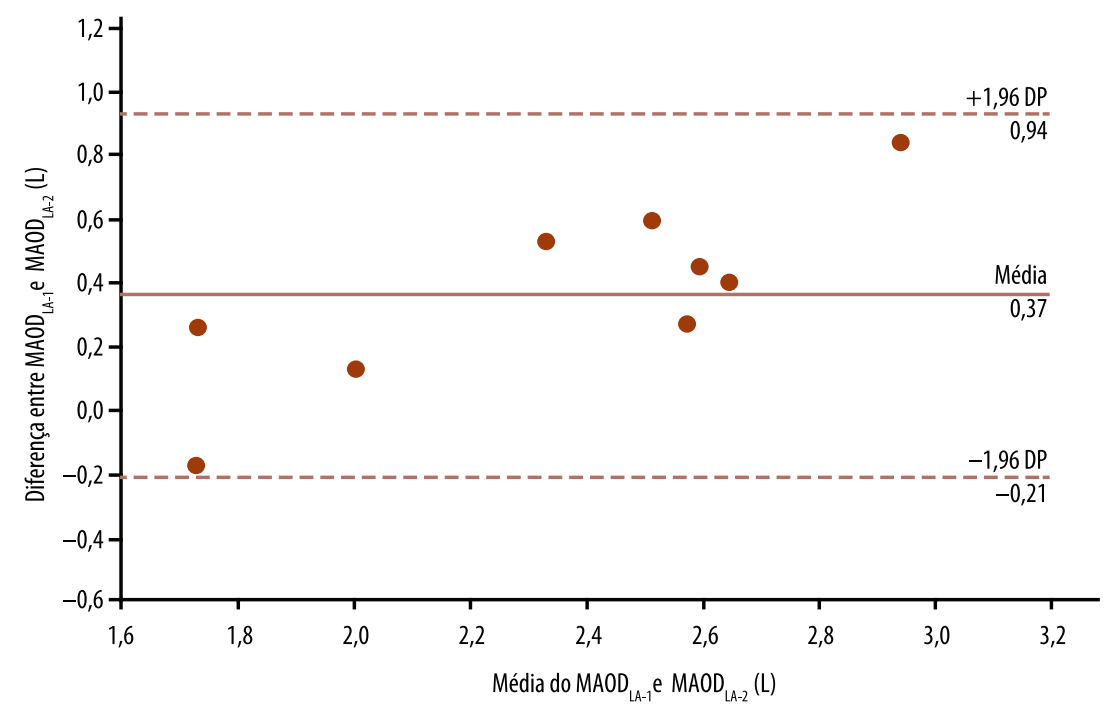

Figura 2. Plotagem de Bland-Altman para a verificação de concordância entre a estimativa do $M A O D_{L A-1}$ e do $M A O D_{L A-2}$

A participação em termos percentuais do $\mathrm{MAOD}_{\mathrm{LA}-1}$ (representando a participação do MAL) e do EPOC $_{\text {RÁPIDO }}$ (representando a participação do MAA) no MAOD foi de 78\% e 22\%, respectivamente. A tabela 3 demonstra os resultados dos termos da equação utilizada para ajustar a resposta da cinética off do $\mathrm{VO}_{2}$.

Tabela 3. Resposta da cinética off do consumo de oxigênio no exercício executado na intensidade de $110 \%$ da WV02max.

\begin{tabular}{lc}
\hline $\mathrm{A}_{1}\left(1 \cdot \mathrm{min}^{-1}\right)$ & $1,35 \pm 0,32$ \\
$\tau_{1}(\mathrm{~s})$ & $28 \pm 6$ \\
$\delta(\mathrm{s})$ & $13 \pm 3$ \\
$\mathrm{~A}_{2}\left(1 \cdot \mathrm{min}^{-1}\right)$ & $0,51 \pm 0,13$ \\
$\tau_{2}(\mathrm{~s})$ & $53 \pm 10$ \\
\hline
\end{tabular}

Os valores são expressos em médias \pm desvios padrão. $A=$ amplitude; $t=$ constante de tempo; 1 e 2 denotam a fase rápida e lenta da reposta cinética off, respectivamente; $d=$ tempo de atraso. 


\section{DISCUSSÃO}

Pelo nosso conhecimento, esse é o primeiro estudo que se propôs analisar a possibilidade de se estimar o componente lático do MAOD mediante a subtração do EPOC $_{\text {RÁPIDO }}$ do MAOD. O principal achado foi que os dois métodos $\left(\mathrm{MAOD}_{\mathrm{LA}-1}\right.$ e $\left.\mathrm{MAOD} \mathrm{LA}-2_{2}\right)$ empregados na estimativa da contribuição do MAL no MAOD geraram valores estatisticamente similares. Além disso, os valores percentuais do $\mathrm{MAOD}_{\mathrm{LA}-1}$ (representando o MAL) e do $\mathrm{EPOC}_{\text {RÁPIDO }}$ (representando o MAA) foram de, aproximadamente, 78\% e $22 \%$, respectivamente.

Entre as limitações dos métodos utilizados para quantificar a contribuição do metabolismo anaeróbio no presente estudo, pode-se destacar o surgimento do componente lento do $\mathrm{VO}_{2}$ max nos testes submáximos que poderia influenciar na estimativa da demanda de oxigênio do exercício supra- $\mathrm{WVO}_{2} \max ^{24}$. Adicionalmente, a contribuição do metabolismo anaeróbio lático pode ser subestimada quando determinada pela concentração de lactato sanguíneo, visto que o lactato muscular pode ser removido por fibras oxidativas antes de entrar na circulação ${ }^{25}$. Independentemente das vantagens e das limitações ${ }^{6-7}$, o MAOD tem sido considerado o procedimento mais aceitável para avaliação indireta da CAN desde o início da década de $1990^{\circ}$. Sua validade tem sido estabelecida a partir de diferentes perspectivas, tais como, a capacidade de acompanhar as mudanças proporcionadas pelo treinamento predominantemente anaeróbio ${ }^{9}$, nas correlações significativas constatadas com outros testes anaeróbios ${ }^{26} \mathrm{e}$ em virtude de se manter inalterado mediante a situação de hipóxia ${ }^{10}$.

Já foi apresentado na literatura que a participação do MAL no MAOD pode ser estimada a partir da biópsia muscular ${ }^{8}$. Outra possível maneira de se estimar esse componente seria através da mensuração da concentração de lactato, utilizando-se o método sugerido por di Prampero e Ferretti ${ }^{14}$. No entanto, o presente estudo demonstrou que o método $\mathrm{MAOD}_{\mathrm{LA}-1}$ foi capaz de estimar a participação do MAL, utilizando apenas a medida do $\mathrm{VO}_{2}$, à medida que não foi detectada diferença significante entre os métodos $\mathrm{MAOD}_{\mathrm{LA}-1}$ e $\mathrm{MAOD}_{\mathrm{LA}-2}$. Além disso, a alta correlação entre os dois métodos $(\mathrm{r}=0,91, \mathrm{p}<0,01)$ e o valor do viés próximo de zero apresentado pela plotagem de Bland-Altman indicam uma elevada concordância entre os dois métodos propostos. Assim, a mensuração de uma única variável $\left(\mathrm{VO}_{2}\right)$ parece ser o suficiente para se estimar a participação do MAL no MAOD, não sendo necessária a utilização de técnicas mais invasivas, como a biópsia muscular e a coleta de sangue para a mensuração da concentração de lactato. Adicionalmente, nessa circunstância, a medida do $\mathrm{VO}_{2}$ não só permite estimar a participação do MAL como também a do MAA, através do cálculo do EPOC $_{\text {RÁPIDO }}$.

Estudos prévios detectaram o aumento de, aproximadamente, $10 \%$ no MAOD após a suplementação com a creatina monohidratada ${ }^{11}$ ou com a cafeína ${ }^{12}$, sugerindo-se que os principais efeitos ergogênios dessas substâncias estão no acréscimo da contribuição do MAA e do MAL, respectivamente. 
No entanto, essas inferências foram realizadas sem a devida obtenção de dados experimentais, haja vista que a versão tradicional do MAOD não permite estimar os seus componentes anaeróbios. Na presente investigação, os dados obtidos acerca das contribuições percentuais do MAL (78\%) e do MAA (22\%) no MAOD são similares aos resultados obtidos por meio de biópsia muscular ${ }^{7}$ ou por estimativas feitas com base em dados das mudanças metabólicas no músculo esquelético descritos na literatura científica ${ }^{10}$. Nesse sentido, parece que a adição do EPOC $_{\text {RÁPIDO }}$ nos cálculos do MAOD pode auxiliar futuros estudos, à medida que eles viabilizam valores percentuais satisfatórios das contribuições dos componentes anaeróbios durante o exercício de alta intensidade.

Alterações no valor do MAOD também são observadas após períodos de treinamento físico ${ }^{9,27}$. Por exemplo, no estudo de Ravier et al. ${ }^{27}$, um grupo de atletas de Karatê realizou o treinamento normal da modalidade e mais duas sessões semanais de treinamento intermitente, composto por 7-9 séries de sprints de 20 segundos (intensidade aproximada de $140 \%$ do $\mathrm{VO}_{2} \max$ ), intercalados por 15 segundos de pausa, enquanto que um outro grupo de atletas da mesma modalidade realizou apenas o treinamento normal de Karatê. Nessa ocasião, verificou-se um aumento de 10,3\% do MAOD no grupo que realizou as duas sessões adicionais de treinamento intermitente após um período de 7 semanas, enquanto que o outro grupo não obteve alterações significantes. Dessa maneira, a investigação da participação do MAA e do MAL no MAOD poderia ser uma estratégia interessante a fim de avaliar qual desses dois sistemas contribuiu mais para a alteração desse índice. Adicionalmente, essa estratégia pode ser interessante, também, nos casos em que se comparam dois tipos diferentes de treinamento ${ }^{28}$, de modo que, mesmo não havendo alterações no valor do MAOD, não exclui a possibilidade de ocorrer alterações significantes na participação relativa dos componentes anaeróbios.

Os valores do MAOD obtidos no presente estudo são similares aos descritos para sujeitos saudáveis em cicloergômetro 9 . Além disso, o MAOD, o tempo de permanência e a potência externa utilizada no teste supramáximo foram substancialmente menores que os reportados para ciclistas profissionais ${ }^{26}$. De certa forma, esses dados corroboram outros achados que demonstraram a capacidade do MAOD em diferenciar sujeitos com diferentes níveis de aptidão anaeróbia ${ }^{29}$.

Em relação à resposta da cinética off do $\mathrm{VO}_{2}$, os resultados obtidos na presente investigação corroboram parcialmente os dados apresentados na literatura científica. Interessantemente, o d foi diferente de zero na intensidade de $110 \% \mathrm{WVO}_{2}$ max. Esse achado confirma os resultados de outro estudo que detectou o d de aproximadamente $25 \mathrm{~s}$ na resposta cinética off do $\mathrm{VO}_{2}$ na corrida intensa $(18 \mathrm{~km} / \mathrm{h} \text { com } 20 \% \text { de inclinação })^{30}$. Isso poderia indicar a existência de uma fase cardiodinâmica ao término do exercício físico exaustivo de curta duração. Em outras palavras, talvez o elevado débito cardíaco induzido pela atividade física intensa prévia seja um dos responsáveis pelo $\mathrm{VO}_{2}$ elevado durante os segundos iniciais da recuperação. 


\section{CONCLUSÕES}

Os achados do presente estudo sugerem que o componente anaeróbio lático do MAOD pode ser estimado satisfatoriamente subtraindo-se o EPOC $_{\text {RÁPIDO }}$ do MAOD. Além disso, o método proposto é capaz de indicar a participação percentual dos metabolismos anaeróbio lático e alático.

\section{REFERÊNCIAS BIBLIOGRÁFICAS}

1. Spencer MR, Gastin PB. Energy system contribution during 200- to 1500-m running in highly trained athletes. Med Science Sports Exerc 2001;33(1):157-62.

2. Gastin PB. Energy system interaction and relative contribution during maximal exercise. Sports Med 2001;31(10):725-41.

3. Gastin PB. Quantification of anaerobic capacity. Scand J Med Sciei Sports 1994;4:91-112.

4. Spriet LL. Anaerobic metabolism during high-intensity exercise. In: Hargreaves M, editor. Exercise metabolism. Champaign: Humans Kinetics; 1995. p. 1-39.

5. Pripstein LP, Rhodes EC, McKenzie DC, Coutts KD. Aerobic and anaerobic energy during a 2-km race simulation in female rowers. Eur J Appl Physiol 1999;79(6):491-4.

6. Bangsbo J. Oxygen deficit: a measure of the anaerobic energy production during intense exercise? Can J Appl Physiol 1996;21(5):350-363.

7. Buck D, McNaughton LR. Changing the number of submaximal exercise bouts effects calculation of MAOD Int J Sports Med 1999;20(1):28-33.

8. Medbø JI, Tabata I. Anaerobic energy release in working muscle during $30 \mathrm{~s}$ to 3 min of exhausting bicycling J Appl Physiol 1993;75(4):1654-60.

9. Weber CL, Schneider DA. Increases in maximal accumulated oxygen deficit after high-intensity interval training are not gender dependent. J Appl Physiol 2002;92(5):1795-801.

10. Medbø JI, Mohn AC, Tabata I, Bahr R, Vaage O, Sejersted OM. Anaerobic capacity determined by maximal accumulated O2 deficit. J Appl Physiol 1998;64(1):50-60.

11. Jacobs I, Bleue S, Goodman J. Creatine ingestion increases anaerobic capacity and maximum accumulated oxygen déficit. Can J Appl Physiol 1997;22(3)231-43.

12. Doherty $M$. The effects of caffeine on the maximal accumulated oxygen deficit and short-term running performance. Int J Sport Nutr 1998;8(2)95-104.

13. Bogdanis GC, Nenill ME, Lakomy HK, Boobis LH. Power output and muscle metabolism during and following recovery from 10 and $20 \mathrm{~s}$ of maximal sprint exercise in humans. Acta Physiol Scand 1998;163(3):261-72.

14. di Prampero PE, Ferretti G. The energetics of anaerobic muscle metabolism: a reappraisal of older and recent concepts. Respir Physiol 1999;118:103-115.

15. Bertuzzi RCM, Franchini E, Kokubun E, Kiss MADM. Energy system contributions in indoor rock climbing. Eur J Appl Physiol 2007;101(3):293-300.

16. Beneke R, Beyer T, Jachner C, Erasmus J, Hütler M. Energetics of karate kumite. Eur J Appl Physiol 2004;92(4-5):518-23.

17. Norton K, Olds T. Antropometrica. Sidney: Southwood Press; 1996.

18. Jackson AS, Pollock ML. Practical assessment of body composition. Phys Sports Med 1985;13:76-90.

19. Brozek J, Grande F, Anderson J, Keys A. Densitometric analysis of body composition: revision of some quantitative assumptions. Ann N Y Acad Scie 1963;110:113-40.

20. Howley ET, Basset DT, Welch HG. Criteria for maximal oxygen uptake: review and commentary. Med Scie Sports Exerc 1995;27(9):1292-301.

21. Mezzani A, Corra U, Sassi B, Colombo R, Giordano A, Giannuzzi P. Maximal accumulated oxygen deficit in patients with chronic heart failure. Med Scie Sports Exer 2006;38(3):424-32.

22. Özyener F, Rossiter HB, Ward SA, Whipp BJ. Influence of exercise intensity on the on- and off-transient kinetics of pulmonary oxygen uptake in humans. J Physiol 2001;533(3):891-902. 
23. Bland JM, Altman DG. Statistical methods of assessing agreement between two methods of clinical measurements. Lancet 1986;1:307-310.

24. Bangsbo J. Is the $\mathrm{O} 2$ deficit an accurate quantitative measure of the anaerobic energy production during intense exercise? J Appl Physiol 1992;73(3):1207-9.

25. Gladden LB. A "Lactatic" perspective on metabolism. Med Scie Sports Exerc 2008;40(3):477-485.

26. Scott CB, Rooby FB, Lohman TG, Bunt JC. The maximally accumulated oxygen deficit as an indicator of anaerobic capacity. Med Scie Sports Exerc 1991;23(5):618-24.

27. Ravier G, Dugué B, Grappe F, Rouillon JD. Impressive anaerobic adaptations in elite karate athletes due to few intensive intermittent sessions added to regular karate training. Scand J Med Sci Sports 2009;19(5):687-94.

28. Roberts AD, Clark SA, Townsend NE, Anderson ME, Gore CJ, Hahn AG. Changes in performance, maximal oxygen uptake and maximal accumulated oxygen deficit after 5, 10 and 15 days of live high:train low altitude exposure. Eur J Appl Physiol 2003;88(4-5):390-5.

29. Buck D, McNaughton L. Maximal accumulated oxygen deficit must be calculated using 10-min time periods. Med Scie Sports Exerc 1999;31(9):1346-9.

30. di Prampero PE, Peeters L, Margaria R. Alactic $\mathrm{O} 2$ debt and lactic acid production after exhausting exercise in man. J Appl Physiol 1973;34(5):628-32.
Endereço para correspondência

Rodrigo Poles Urso

Universidade de São Paulo. Escola de

Educação Física e Esporte.

Departamento de Esporte

Av. Prof. Mello Moraes, 65. Cidade

Universitária

05508-030 - São Paulo, SP. Brasil

E-mail: rodrigo.urso@usp.br 